\title{
Association between personal characteristics and musculoskeletal disorders among the dental surgeons working in Dhaka city
}

\author{
Dr. Nihar Sultana ${ }^{1 *}$, Dr. Md.Golam Rubby ${ }^{2}$, Prof. Dr. Md. Anower Hussain Mian ${ }^{3}$.
}

\section{AFFILIATION:}

1. Dr. Nihar Sultana *

Assistant Professor, Department of Dental Anatomy, Mandy Dental College, Dhaka; Bangladesh

PhD Research Fellow (Bangladesh University of Professionals)

2. Dr. Md.Golam Rubby

Associate Professor and Head, Department of Orthodontics, Mandy Dental College, Dhaka. Bangladesh

3. Prof. Dr. Md. Anower Hussain Mian

Professor and Head, Department of Community Dentistry, Dean, Faculty of Public Health,

Bangladesh University of Health Sciences (BUHS).

\section{Article info.}

Received: 12 January 2021

Accepted: 18 March 2021

Volume: Vol-11, Issue-1, April 2021

DOI: https://doi.org/10.3329/updcj.v11i1.53002

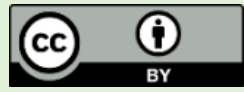

(C) Authors retain copyright and grant the journal right of first publication with the work simultaneously licensed under Creative Commons Attribution License CC - BY 4.0 that allows others to share the work with an acknowledgment of the work's authorship and initial publication in this journal.

https://creativecommons.org/licenses/by/4.0/

Publisher: Update Dental College, Dhaka, Bangladesh

Web: www.updatedentalcollege.edu.bd

E-mail: updcj@hotmail.com

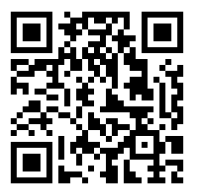

Scan QR code to see the latest issue

* Corresponding Author

Dr. Nihar Sultana

Assistant Professor

Department of Dental Anatomy

Mandy Dental College \& Hospital, Bangladesh

PhD Research Fellow (BUP)

Phone: $+8801712-843965$

E-mail: nihard40@gmail.com

\section{ABSTRACT:}

Background: Occupational hazards are common in different working populations. Musculoskeletal disorders (MSDs) are the most important occupational hazards particularly in dental professionals. This disorder which is multi factorial in origin has relation with different characteristics. The study aims to find out the association between personal characteristics in terms of socio demographic, occupation characteristics and MSDs among the dental surgeons working in Dhaka city.

Methods: Present cross sectional study was conducted among purposively selected 290 dental surgeons working in thirteen private and public tertiary level hospitals in Dhaka city. A structured questionnaire was used for data collection including socio demographic and occupational characteristics which reflect the personal characteristics of dental surgeons. To determine the MSDs, Nordic Musculoskeletal Questionnaire (NMQ) was used which records the prevalence of MSDs in terms of musculoskeletal symptoms. Collected data were analyzed by using SPSS software (Version 22).

Results : Almost $64.0 \%$ dental surgeons experienced MSDs in past 12 months in at least one region of the body, and the major affected part was neck, followed by lower back, shoulders and hands / wrists. Regarding the relationship between personal characteristics and MSDs, present study found significant relationship exists between some socio demographic characteristics like age, physical activity; some occupational characteristics like work status, practicing area, practicing four handed dentistry, maintaining back position upright, psychosocial stress with MSDs in chi square test.

Conclusion: This study concludes that the existence of significant relationship between different personal characteristics and MSDs among dental surgeons which need to be address properly, so that dental professionals will be able to take corrective measures to combat with this disorder.

KEYWORDS: Musculoskeletal disorders; Personal characteristics; Risk; Dental surgeons; Occupational hazards.

\section{INTRODUCTION:}

Occupational hazards are common in different working populations. Musculoskeletal disorders (MSDs) are a significant occupational health hazard that affect members of various occupations. ${ }^{[1]}$ This disorder is characterized by presence of persistent pain ,discomfort or disability in the muscles, joints, nerves, ligaments, tendons, and other soft parts of the body, caused or aggravated by repeated movements and forced body postures or prolonged awkward body postures. ${ }^{[2]}$ Dental professionals are at risk of developing this disorder because of using different vibratory tools during wok, excessive repetitive movements, maintaining a static position while performing extremely precise different dental procedures in a small workspace, and/or maintaining an inadequate posture for long periods of time. It has been noted that dentists have a higher prevalence of MSDs compared with office workers. ${ }^{[3]}$ The literature on MSDs among dental professionals indicates that these complaints have contributed considerably to morbidity, as well as reducing productivity and quality of work and possibly leading to premature retirement. ${ }^{[4-5]}$ Further to the point, the symptoms of MSDs increase with the number of years of practice. ${ }^{[6-7]}$ In our country the prevalence of MSDs in dentistry is not well documented, as well as the existence of relationship of different characteristics with this disorder is not explored. The present study focused on association between personal characteristics and musculoskeletal disorders among the dental surgeons working in Dhaka city. 


\section{MATERIALS AND METHODS :}

Present cross sectional study conducted among purposively selected 290 dental surgeons working in thirteen private and public hospitals in Dhaka city to find out the association between personal characteristics in terms of socio demographic, occupation characteristics and MSDs among them. Considering each study place as a strata data were collected from those study hospitals proportionately according to the convenient sampling technique. The dental surgeons who practice at least 20 hours of clinical work in a week with having at least 5 years of clinical experience were included in this study, and those who had history of any accident or trauma, uncontrolled diabetes, which may influence the musculoskeletal system were excluded from this study. Ethical clearance was obtained from the institutional ethical committee of Bangladesh University of Professionals (BUP) and the reference number was 24.786.51. Before commencing this study a pilot study was conducted among ten dental surgeons.

Prior taking permission from the concerned authority of each study place informing the purpose of the study data were collected from the respondents after taking informed consent by using the interview administered questionnaire including socio-demographic and occupational characteristics which combined as personal characteristics of the respondents in this study. For the analysis of MSDs, Nordic Musculoskeletal Questionnaire (NMQ) was used which records the prevalence of MSDs in terms of musculoskeletal symptoms such as discomfort, pain, ache in the preceding 12 months. ${ }^{[8]}$ It is an internationally accepted instrument to standardize evaluation of MSDs, and consists of questions about nine anatomical regions. Subjects were asked to report whether they had experienced any kind of discomfort or pain in any of these nine regions of the body during the last 12 months or 7 days and whether normal activities were restricted because of these symptoms. To assess the level of knowledge regarding optimal healthy posture among the dental surgeons, a questionnaire developed based on the document of "Adopting a healthy sitting working posture during patient treatment" , integral part of ISO standardization [9] and the categorization of psychosocial stress performed by the psycho-social stress evaluation sheet (Health evaluation questionnaire for psychosocial stress in healthcare industry). Finally collected data were analyzed by using SPSS software (Version 22) and presented in tables and figures by using Microsoft word and excel application. Statistical significance was accepted for values of $p<0.05$.

\section{RESULTS :}

Table 1 : Descriptive statistics of personal characteristics

\begin{tabular}{|c|c|}
\hline Variables & Participants ( $\mathrm{N}=\mathbf{2 9 0})$ \\
\hline Age (in years) $M \pm S D$ & $36.1 \pm 5.7$ \\
\hline BMI $M \pm S D$ & $25.06 \pm 3.36$ \\
\hline Professional experience (in years) $\mathrm{M} \pm \mathrm{SD}$ & $12.9 \pm 5.3$ \\
\hline Practicing hours (per week) $M \pm S D$ & $45.6 \pm 13.4$ \\
\hline Patients attended (per day) $\mathrm{M} \pm \mathrm{SD}$ & $10 \pm 4.7$ \\
\hline Smoking $\mathrm{n}(\%)$ & $42(22.6)$ \\
\hline Regular physical activity $n$ (\%) & $67(36.0)$ \\
\hline Full time work status in last 12 months $n(\%)$ & $141(75.8)$ \\
\hline \multicolumn{2}{|c|}{$\begin{array}{l}\text { M - mean , SD - standard deviation } \\
\text { Above table shows the descriptive statistics of different personal characteristics of the } \\
\text { study subjects. The average age of the study subjects was } 36.1 \pm 5.7 \text { years, the mean } \\
\text { orofessional experience was } 12.9 \pm 5.3 \text { years, } 22.6 \% \text { respondents were smoker, } 75.8 \% \\
\text { of the respondents work status was full time (both evening and morning shift) in the last } \\
12 \text { months. }\end{array}$} \\
\hline
\end{tabular}

12| $\mathrm{P}$ a g $\mathrm{e}$
Figure 1: Prevalence of musculoskeletal disorders at any region of the body among the dental surgeons in last 12 months

$[\mathrm{n}=\mathbf{2 9 0}$ ]

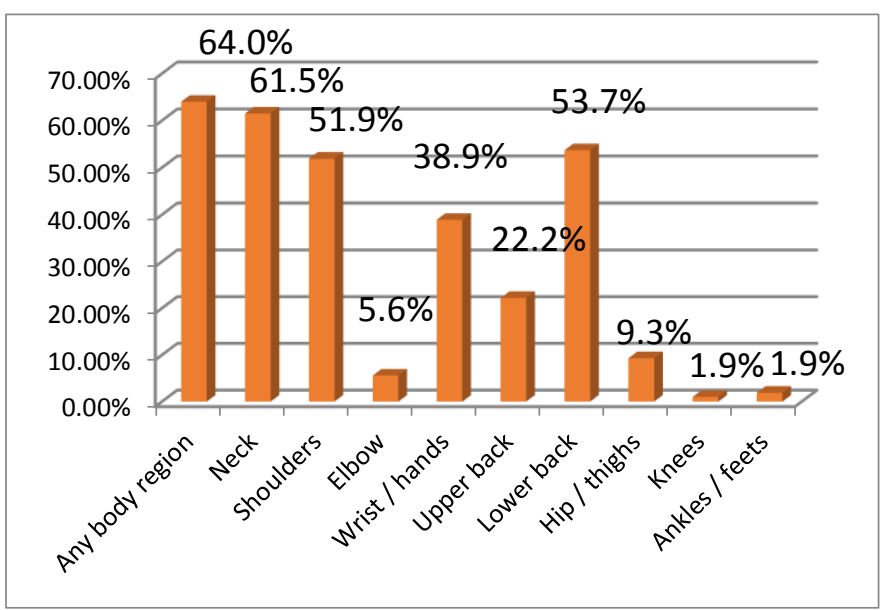

Out of $290(100.0 \%)$ dental surgeons $64.0 \%$ had complaints of MSDs at any body region in last 12 months. The highest complained region was neck (61.5\%), followed by lower back (53.7\%), shoulders $(51.9 \%)$, hands/ wrists $(38.9 \%)$ and the least complained region was ankles/feet, knees $(1.9 \%)$ in the last 12 months

Table 2: Association between the socio demographic characteristics with MSDs among the dental surgeons

\begin{tabular}{|c|c|c|c|c|c|}
\hline $\begin{array}{c}\text { Socio } \\
\text { demographic } \\
\text { characteristics }\end{array}$ & Category & $\begin{array}{c}\text { Total } \\
(n=290) \%\end{array}$ & $\begin{array}{l}\text { Without } \\
\text { MSDs } \\
n(\%)\end{array}$ & $\begin{array}{l}\text { With } \\
\text { MSDs } \\
\mathrm{n}(\%)\end{array}$ & $\begin{array}{l}\mathrm{p} \\
\text { value }\end{array}$ \\
\hline \multirow[t]{4}{*}{ Age (in years) } & $\leq 32$ & $87(30.0)$ & $35(33.7)$ & $\begin{array}{c}52 \\
(28.0)\end{array}$ & \multirow{4}{*}{$.035^{\mathrm{s}}$} \\
\hline & $33-37$ & $111(38.3)$ & $49(47.1)$ & $\begin{array}{c}62 \\
(33.3)\end{array}$ & \\
\hline & $38-42$ & $63(21.7)$ & $11(10.6)$ & $\begin{array}{c}52 \\
(28.0)\end{array}$ & \\
\hline & $>42$ & $29(10.0)$ & $9(8.7)$ & $\begin{array}{c}20 \\
(10.8)\end{array}$ & \\
\hline \multirow[t]{2}{*}{ Sex } & Male & $182(62.8)$ & $56(53.8)$ & $\begin{array}{c}126 \\
(67.7)\end{array}$ & \multirow[t]{2}{*}{$.152^{\mathrm{ns}}$} \\
\hline & Female & $108(37.2)$ & $48(46.2)$ & $\begin{array}{c}60 \\
(32.3)\end{array}$ & \\
\hline \multirow[t]{2}{*}{ BMI } & $\begin{array}{l}\text { Underweight } \\
\text { \& normal }\end{array}$ & $141(48.6)$ & $56(53.8)$ & $\begin{array}{c}85 \\
(45.7)\end{array}$ & \multirow[t]{2}{*}{$.183^{n}$} \\
\hline & $\begin{array}{c}\text { Overweight \& } \\
\text { obese }\end{array}$ & $149(51.4)$ & $48(46.2)$ & $\begin{array}{c}101 \\
(54.3)\end{array}$ & \\
\hline \multirow[t]{2}{*}{ Physical activity } & Yes & $120(41.4)$ & $53(51.0)$ & $\begin{array}{c}67 \\
(36.0)\end{array}$ & \multirow[t]{2}{*}{$.028^{\mathrm{s}}$} \\
\hline & No & $170(58.6)$ & $51(49.0)$ & $\begin{array}{c}119 \\
(64.0)\end{array}$ & \\
\hline \multirow[t]{2}{*}{ Smoking } & Yes & $48(16.6)$ & $6(5.8)$ & $\begin{array}{c}42 \\
(22.6)\end{array}$ & \multirow[t]{2}{*}{$.07^{\mathrm{ns}}$} \\
\hline & No & $242(83.4)$ & $98(94.2)$ & $\begin{array}{c}144 \\
(77.4)\end{array}$ & \\
\hline
\end{tabular}

p-value was calculated by chi square test ,hs: Highly significant, s : Significant, ns : Not significant p-value significant at $<0.05$

Above table shows association between socio demographic characteristics and MSDs among the dental surgeons. Age, physical activity had significant association with MSDs, (as $p$ value $<0.05$ ). No statistical significant association found between other socio demographic characteristics like sex, BMI, smoking with MSDs (as p value <0.05).

Website: https://www.banglajol.info/index.php/UpDCJ 
Table 3: Association between job related characteristics with MSDs among dental surgeons

\begin{tabular}{|c|c|c|c|c|c|}
\hline $\begin{array}{c}\text { Job related } \\
\text { characteristics }\end{array}$ & Category & $\begin{array}{c}\text { Total } \\
(\mathrm{n}=290) \\
\%\end{array}$ & $\begin{array}{c}\text { Without } \\
\text { MSDs n } \\
(\%)\end{array}$ & $\begin{array}{l}\text { With } \\
\text { MSDs } \\
\mathrm{n}(\%)\end{array}$ & $\begin{array}{l}\mathrm{p} \\
\text { value }\end{array}$ \\
\hline \multirow{4}{*}{$\begin{array}{l}\text { Professional } \\
\text { experience (in } \\
\text { years) }\end{array}$} & $\leq 8$ & $54(18.6)$ & $\begin{array}{c}20 \\
(19.2)\end{array}$ & $\begin{array}{c}34 \\
(18.3)\end{array}$ & \multirow{4}{*}{$.122^{\text {ns }}$} \\
\hline & $9-13$ & $\begin{array}{c}135 \\
(46.6)\end{array}$ & $\begin{array}{c}58 \\
(55.8)\end{array}$ & $\begin{array}{c}77 \\
(41.4)\end{array}$ & \\
\hline & $14-18$ & $69(23.8)$ & $\begin{array}{c}17 \\
(16.3)\end{array}$ & $\begin{array}{c}52 \\
(28.0)\end{array}$ & \\
\hline & $>18$ & $32(11.0)$ & $9(8.7)$ & $\begin{array}{c}23 \\
(12.4)\end{array}$ & \\
\hline \multirow[t]{2}{*}{$\begin{array}{l}\text { Working } \\
\text { hospital }\end{array}$} & $\begin{array}{c}\text { Public or } \\
\text { autonomous }\end{array}$ & 179(61.7) & $\begin{array}{c}70 \\
(67.3)\end{array}$ & $\begin{array}{c}109 \\
(58.6)\end{array}$ & \multirow[t]{2}{*}{$\begin{array}{c}.144 \\
\text { ns }\end{array}$} \\
\hline & Private & $\begin{array}{c}111 \\
(38.3)\end{array}$ & $\begin{array}{c}34 \\
(32.7)\end{array}$ & $\begin{array}{c}77 \\
(41.4)\end{array}$ & \\
\hline \multirow[t]{2}{*}{$\begin{array}{c}\text { Work status } \\
\text { in past } 12 \\
\text { months }\end{array}$} & $\begin{array}{l}\text { Full time } \\
\text { (Both } \\
\text { evening and } \\
\text { morning } \\
\text { shift) }\end{array}$ & $\begin{array}{c}179 \\
(61.7)\end{array}$ & $\begin{array}{c}38 \\
(36.5)\end{array}$ & $\begin{array}{c}141 \\
(75.8)\end{array}$ & \multirow[t]{2}{*}{$\begin{array}{c}.000 \\
\text { hs }\end{array}$} \\
\hline & $\begin{array}{l}\text { Part time } \\
\text { (Only } \\
\text { morning or } \\
\text { evening) }\end{array}$ & $\begin{array}{c}111 \\
(38.3)\end{array}$ & $\begin{array}{c}66 \\
(63.5)\end{array}$ & $\begin{array}{c}45 \\
(24.2)\end{array}$ & \\
\hline \multirow[t]{3}{*}{$\begin{array}{l}\text { Current area } \\
\text { of practice }\end{array}$} & Clinical & $\begin{array}{c}257 \\
(88.6)\end{array}$ & $\begin{array}{c}84 \\
(80.4)\end{array}$ & $\begin{array}{c}173 \\
(93.0)\end{array}$ & \multirow{3}{*}{$\begin{array}{c}.004 \\
\text { hs }\end{array}$} \\
\hline & $\begin{array}{c}\text { Academic / } \\
\text { Public } \\
\text { health }\end{array}$ & $21(7.2)$ & $\begin{array}{c}14 \\
(13.5)\end{array}$ & $7(3.8)$ & \\
\hline & $\begin{array}{l}\text { Clinical \& } \\
\text { academic }\end{array}$ & $4(4.7)$ & $2(6.5)$ & $2(3.6)$ & \\
\hline \multirow[t]{3}{*}{$\begin{array}{l}\text { Nature of } \\
\text { practice }\end{array}$} & General & $48(16.6)$ & $\begin{array}{c}21 \\
(20.2)\end{array}$ & $\begin{array}{c}27 \\
(14.5)\end{array}$ & \multirow[t]{3}{*}{.245} \\
\hline & Specialized & $54(18.6)$ & $\begin{array}{c}22 \\
(21.2)\end{array}$ & $\begin{array}{c}32 \\
(17.2)\end{array}$ & \\
\hline & Both & $\begin{array}{c}188 \\
(64.8)\end{array}$ & $\begin{array}{c}61 \\
(58.7)\end{array}$ & $\begin{array}{c}127 \\
(68.3)\end{array}$ & \\
\hline \multirow[t]{2}{*}{$\begin{array}{l}\text { Involve in } \\
\text { teaching }\end{array}$} & Yes & $\begin{array}{c}102 \\
(35.2)\end{array}$ & $\begin{array}{c}34 \\
(32.7)\end{array}$ & $\begin{array}{c}68 \\
(36.6)\end{array}$ & \multirow[t]{2}{*}{$\begin{array}{c}.508 \\
\text { ns }\end{array}$} \\
\hline & No & $188(64.8)$ & $\begin{array}{c}70 \\
(67.3)\end{array}$ & $\begin{array}{c}118 \\
(63.4)\end{array}$ & \\
\hline
\end{tabular}

p-value was calculated by chi square test,

hs: Highly significant, s : Significant, ns :Not significant,

p-value significant at $<0.05$

Above table shows association between job related characteristics and MSDs among the dental surgeons. Here strong statistical significant association found between work status in last 12 months with MSDs (as $p$ value $<0.01$ ) and current area of practice with MSDs (as $p$ value $<0.01$ ) . No statistical significant association found between other job related characteristics like professional experience, working hospital, area of practice, involvement in teaching with MSDs ( as $p$ value $<.05$ ).

13| P a g e
Table 4: Association between practice related variables with MSDs among

\begin{tabular}{|c|c|c|c|c|c|}
\hline $\begin{array}{l}\text { Practice related } \\
\text { variables }\end{array}$ & Category & $\begin{array}{c}\text { Total } \\
\mathrm{n}=290 \\
(\%)\end{array}$ & $\begin{array}{l}\text { Without } \\
\text { MSDs } \\
n(\%)\end{array}$ & $\begin{array}{l}\text { With } \\
\text { MSDs } \\
\mathrm{n}(\%)\end{array}$ & $\begin{array}{l}\mathrm{p} \\
\text { value }\end{array}$ \\
\hline \multirow{2}{*}{$\begin{array}{l}\text { Using indirect } \\
\text { vision (where } \\
\text { needed) }\end{array}$} & No & $\begin{array}{c}125 \\
(43.1)\end{array}$ & $23(22.1)$ & $\begin{array}{c}102 \\
(54.8)\end{array}$ & \multirow[t]{2}{*}{.000 hs } \\
\hline & Yes & $\begin{array}{c}165 \\
(56.9)\end{array}$ & $81(77.9)$ & $\begin{array}{c}84 \\
(45.2)\end{array}$ & \\
\hline \multirow{2}{*}{$\begin{array}{l}\text { Used to bend } \\
\text { for more } \\
\text { visibility }\end{array}$} & No & $\begin{array}{c}146 \\
(50.3)\end{array}$ & $78(75.0)$ & $\begin{array}{c}68 \\
(36.6)\end{array}$ & \multirow[t]{2}{*}{.000 hs } \\
\hline & Yes & $\begin{array}{c}144 \\
(49.7)\end{array}$ & $26(25.0)$ & $\begin{array}{c}118 \\
(63.4)\end{array}$ & \\
\hline \multirow{2}{*}{$\begin{array}{l}\text { Used to } \\
\text { maintain back } \\
\text { position } \\
\text { upright }\end{array}$} & No & $12(4.1)$ & $6(5.8)$ & $6(3.2)$ & \multirow[t]{2}{*}{$.012^{\mathrm{hs}}$} \\
\hline & Yes & $\begin{array}{c}278 \\
(95.9)\end{array}$ & $98(94.2)$ & $\begin{array}{c}180 \\
(96.8)\end{array}$ & \\
\hline \multirow{2}{*}{$\begin{array}{l}\text { Using } \\
\text { magnifying } \\
\text { loupes }\end{array}$} & No & $\begin{array}{c}278 \\
195.9\end{array}$ & $95(91.3)$ & $\begin{array}{c}183 \\
(98.4)\end{array}$ & \multirow[t]{2}{*}{$.125^{\mathrm{ns}}$} \\
\hline & Yes & $12(4.1)$ & $9(8.7)$ & $3(1.6)$ & \\
\hline \multirow{2}{*}{$\begin{array}{l}\text { Frequently get } \\
\text { up from work } \\
\text { or change } \\
\text { posture }\end{array}$} & No & $18(6.2)$ & $3(2.9)$ & $15(8.1)$ & \multirow[t]{2}{*}{$.043^{s}$} \\
\hline & Yes & $\begin{array}{c}272 \\
(93.8)\end{array}$ & 101 (97.1) & $\begin{array}{c}171 \\
(91.9)\end{array}$ & \\
\hline \multirow{2}{*}{$\begin{array}{l}\text { Practice four } \\
\text { handed or six } \\
\text { handed } \\
\text { dentistry }\end{array}$} & No & $6(2.1)$ & $0(0)$ & $6(3.2)$ & \multirow[t]{2}{*}{$.035^{s}$} \\
\hline & Yes & $\begin{array}{c}284 \\
(97.9)\end{array}$ & $\begin{array}{c}104 \\
(100.0)\end{array}$ & $\begin{array}{c}180 \\
(96.8)\end{array}$ & \\
\hline
\end{tabular}

p-value was calculated by chi square test

hs: Highly significant, s : Significant, ns :Not significant p-value significant at $<\mathbf{0 . 0 5}$

Above table shows the association between different practice related variables with MSDs. Here the practice related variables; using indirect vision, used to bend for more visibility, maintaining back position upright had highly significant association with musculoskeletal disorders (as $p$ value less than 0.01). Frequently get up from work or change posture and practicing four handed or six handed dentistry had significant association with musculoskeletal disorders (as $p$ value less than 0.05 ); where as using magnifying loupes was not significantly associated with MSDs.

Table 5: Association between knowledge regarding optimal healthy posture and psychosocial stress with MSDs among dental surgeons

\begin{tabular}{|c|c|c|c|c|c|}
\hline Variables & Category & $\begin{array}{c}\text { Total } \\
(n=290) \\
\%\end{array}$ & $\begin{array}{c}\text { Without } \\
\text { MSDs } \\
\mathrm{n}(\%)\end{array}$ & $\begin{array}{c}\text { With } \\
\text { MSDs } \\
\mathrm{n}(\%)\end{array}$ & $\begin{array}{l}\mathrm{p} \\
\text { value }\end{array}$ \\
\hline \multirow{3}{*}{$\begin{array}{l}\text { Knowledge } \\
\text { regarding } \\
\text { optimal healthy } \\
\text { posture }\end{array}$} & Poor & $12(4.1)$ & $0(0)$ & $12(6.5)$ & \multirow{3}{*}{$.065 \mathrm{~ns}$} \\
\hline & Average & $78(26.9)$ & $8(7.7)$ & $\begin{array}{c}70 \\
(37.6)\end{array}$ & \\
\hline & Good & $\begin{array}{c}200 \\
(69.0)\end{array}$ & $96(92.3)$ & $\begin{array}{c}104 \\
(55.9)\end{array}$ & \\
\hline \multirow[t]{3}{*}{$\begin{array}{l}\text { Psychosocial } \\
\text { stress }\end{array}$} & Low & $\begin{array}{c}129 \\
(44.5)\end{array}$ & 79 (76.0) & $\begin{array}{c}50 \\
(26.9)\end{array}$ & \multirow[t]{3}{*}{.000 hs } \\
\hline & Moderate & $\begin{array}{c}143 \\
(49.3)\end{array}$ & $19(18.3)$ & $\begin{array}{c}124 \\
(66.7)\end{array}$ & \\
\hline & High & $18(6.2)$ & $6(5.8)$ & $12(6.5)$ & \\
\hline
\end{tabular}

p-value was calculated by chi square tesths: Highly significant, s : Significant, ns :Not significant, $\mathrm{p}$-value significant at $<0.05$

Above table shows, there was no statistical significant association between knowledge regarding optimal healthy posture among the dental surgeons with MSDs ( as $p$ value > $0.05)$. But highly significant association found in between psychosocial stress and MSDs (as $p$ value $<0.01$ )

Website: https://www.banglajol.info/index.php/UpDCJ 
Update Dental College Journal (UpDCJ): Vol- 11, Issue-1

Table 6: Relationship between personal characteristics and MSDs among dental surgeons

Personal characteristics $\quad p$ value $\quad$ Relationship with

MSDs

\begin{tabular}{|c|c|c|}
\hline Age* $^{*}$ & .035 & Exists \\
\hline Sex & .152 & Does not exist \\
\hline BMI & .183 & Does not exist \\
\hline Physical activity* & .028 & Exists \\
\hline Professional experience & .122 & Does not exist \\
\hline Work status in the past 12 months* & .000 & Exists \\
\hline Area of practice* & .004 & Exists \\
\hline Nature of practice & .245 & Does not exist \\
\hline Practicing four handed dentistry* & .035 & Exists \\
\hline Maintaining back position upright ${ }^{*}$ & .012 & Exists \\
\hline Psychosocial stress* & .000 & Exists \\
\hline Knowledge regarding optimal healthy & .065 & Does not exist \\
\hline
\end{tabular}

posture

* significant

p-value was calculated by chi square test

p-value significant at $<0.05$

Above table shows the relationship exists between the personal characteristics such as age, physical activity, work status, area of practice, practicing four handed dentistry, back position maintaining, psychosocial stress with MSDs; where as with other personal characteristics like sex, BMI, professional experience, nature of practice, knowledge regarding optimal healthy posture does not exists any relationship with MSDs.

DISCUSSION

The prevalence of MSDs among dental professionals from various research studies has found to be higher. The present study focused on the relationship between personal characteristics in terms of socio demographic and occupational characteristics with musculoskeletal disorders among dental surgeons working in Dhaka city. According to present study result $64.0 \%$ dental surgeons experienced MSDs at least one body region in past 12 months, quite higher prevalence observed in at least one body region in the past 12 months in different studies like 79\% ( $n=2166)$ in Gupta et al. ${ }^{[10]}, 83.1 \%(n=213)$ in Saxena et al. ${ }^{[11]}, 86.8 \%$ in KiHun et al. ${ }^{[12]}, 92 \%$ in Batham et al. ${ }^{[13]}$. In addition MSDs in dental surgeons were found in neck region (61.5\%), lower back $(53.7 \%)$, shoulders $(51.9 \%)$, hands/ wrists $(38.9 \%)$ and upper back (22.2\%). Regarding the relationship between personal characteristics and MSDs, present study showed significant relationship exists between some socio demographic characteristics like age, physical activity; some occupational characteristics like work status, practicing area, practicing four handed dentistry, maintaining back position upright, psychosocial stress with MSDs in chi square test. In addition present study found significant association between age and MSDs, which supports the study results of Cromie et al..14], but inconsistent with the study results by Tinubu et al..[15], there were no statistically significant differences in terms of sex, BMI, smoking between with MSDs and without MSDs group, which was consistent with the study results ${ }^{[16,17]}$ and inconsistent in case of BMI with the study ${ }^{[13]}$. No statistically significant differences observed in terms of job related characteristics like professional experiences between with MSDs and without MSDs group, which supports the study result of Rafie, et al. ${ }^{[17]}$ but highly significant relationship observed between MSDs and work status in last 12 months $(p=.000)$ and also with

14|P a g e current area of practice $(p=004)$. Some practice related varia were strongly associated with MSDs, which were using indirect vision $(p=.000)$, used to bend for more visibility $(p=.000)$, maintaining back position upright $(p=.012)$. Again psychosocial stress was highly associated with MSDs (as p value <.01), which was consistent to the study conducted by KiHun, et al..12]; where they reported that occupational stress was a risk factor for increased MSDs in dental practitioners.

\section{CONCLUSION}

This study concludes that the existence of significant relationship between different personal characteristics and MSDs among dental surgeons which need to be address properly, so that dental professionals will be able to take corrective measures to combat with this disorder.

CONFLICT OF INTEREST: Author declared no conflict of interest

\section{REFERENCES:}

1. Saxena P, Gupta SK, Jain S, Jain D. Work-Related Musculoskeletal Pain Among Dentists in Madhya Pradesh, india: Prevalence, Associated Risk Factors, and Preventive Measures. Asia Pac J Public Health 2014; 26: 304309.

https://doi.org/10.1177/1010539513497784 PMid:24097923

2. Valachi B, Valachi K. Preventing musculoskeletal disorders in clinical dentistry: Strategies to address the mechanisms leading to musculoskeletal disorders. J Am Dent Assoc.2003;134:1604-12 https://doi.org/10.14219/jada.archive.2003.0106 PMid: 14719757

3. Alexandre PC, da Silva iC, de Souza LM, de Magalhães CV, Palacios M Meyer A: Musculoskeletal disorders among Brazilian dentists. Arch environ Occup Health 2011; 66: 231-235.

https://doi.org/10.1080/19338244.2011.564571 PMid:22014196

4. Crawford L, Gutierrez G, Harber P. Work environment and occupational health of dental hygienists: a qualitative assessment. J Occup environ Med 2005; 47: 623-632. https://doi.org/10.1097/01.jom.0000165744.47044.2b PMid: 15951722

5. Leggat PA, Kedjarune U, Smith DR. Occupational health problems in modern dentistry: a review. ind Health 2007; 45: 611-621. https://doi.org/10.2486/indhealth.45.611 PMid:18057804

6. Oliveira Dantas FF, de Lima KC. the relationship between physical load and musculoskeletal com.plaints among Brazilian dentists. Appl ergon 2015; 47: 93-98.

https://doi.org/10.1016/i.apergo.2014.09.003 PMid:25479978

7. Kierklo A, Kobus A, Jaworska M, Botulinski B. Work-related musculoskeletal disorders among dentists-a questionnaire survey. Ann Agric environ Med 2011; 18: 79-84.

8. Kuorinka B, Jonsson A, Kilbom H, Vinterberg F, Biering G, Andersson K Jorgensen. Standardised Nordic questionnaires for the analysis of musculoskeletal symptoms. Applied Ergonomics. 1987; 18.3, 233-237. https://doi.org/10.1016/0003-6870(87)90010-X

9. Adopting a healthy sitting working posture during patient treatment.Optical Ergonomic Solutions, 2006.

10. Gupta D, Batra R, Mahajan S, Bhaskar D J , Jain A , Shiju M , et al. Comparative evaluation of the complementary and alternative medicine therapy and conventional therapy use for musculoskeletal disorders management and its association with job satisfaction among dentists of West India. J Tradit Complement Med . $2014 ; 4: 263-7$ https://doi.org/10.4103/2225-4110.126632 PMid:25379469 PMCid:PMC4220505 
11. Saxena P, Gupta S K, Jain S, Jain D. Work-related musculoskeletal pain among dentists in Madhya Pradesh, India: Prevalence, associated risk factors, and preventive measures. Asia Pac J Public Health .2014; 26:304-9 https://doi.org/10.1177/1010539513497784

PMid:24097923

12. KiHun C, Hwi-young C, Gyeong S H. Risk factors associated with musculoskeletal symptoms in Korean dental practitioners. Journal of Physical Therapy Science . 2016; Jan; 28(1): 56-62. https://doi.org/10.1589/ipts.28.56 PMid:26957728 PMCid:PMC4755974

13. Batham C, Yasobant S. A risk assessment study on work-related musculoskeletal disorders among dentists in Bhopal, India. Indian J Dent Res. 2016;27:236-41

https://doi.org/10.4103/0970-9290.186243

PMid:27411650

14. Cromie JE , Robertson VJ, Best MO. Work-Related Musculoskeletal Disorders in Physical Therapists: Prevalence, Severity, Risks, and $\begin{array}{llll}\text { Responses. } & \text { Phys } \quad \text { Ther. } 2000 & ; 00: 336-51\end{array}$ https://doi.org/10.1093/pti/80.4.336 PMid:10758519

15. Tinubu BM, Mbada C E, Oyeyemi A L, Fabunmi A A. Work-Related Musculoskeletal Disorders among Nurses in Ibadan, South-west Nigeria: A cross-sectional. BMC Musculoskelet Disord. 2010 ;11:12 https://doi.org/10.1186/1471-2474-11-12 PMid:20089139 PMCid:PMC2823665

16. Yasobant S, Rajkumar P. Health of the healthcare professionals: A risk assessment study on work-related musculoskeletal disorders in a tertiary hospital, Chennai, India. Int J Med Public Health. 2015 ;5:189-95 https://doi.org/10.4103/2230-8598.153836

17. Rafie F, Jam A Z, Shahravan A, Raoof M, Eskandarizadeh A. Prevalence of Upper Extremity Musculoskeletal Disorders in Dentists: Symptoms and Risk Factors. Journal of Environmental and Public Health. 2015; Article ID 517346 , 6

pages.

https:/doi.org/10.1155/2015/517346

PMid:26064141 PMCid:PMC4433686

To submit your manuscript, Mail us to : updci@hotmail.com

To see our latest issue, click the following link

https://www.banglajol.info/index.php/UpDCJ 